\title{
How Kieffer Density Matrix Formalism Aids Different Initial Time Steps, Leading to Lorentz Violations, and Breakup of Primordial Black Holes for GW Generation
}

\author{
Andrew Beckwith \\ Physics Department, College of Physics, Chongqing University, Chongqing, China \\ Email: rwill9955b@gmail.com
}

How to cite this paper: Beckwith, A. (2021) How Kieffer Density Matrix Formalism Aids Different Initial Time Steps, Leading to Lorentz Violations, and Breakup of Primordial Black Holes for GW Generation. Journal of High Energy Physics, Gravitation and Cosmology, 7, 1315-1327.

https://doi.org/10.4236/jhepgc.2021.74081

Received: August 3, 2021

Accepted: September 25, 2021

Published: September 28, 2021

Copyright $\odot 2021$ by author(s) and Scientific Research Publishing Inc. This work is licensed under the Creative Commons Attribution International License (CC BY 4.0).

http://creativecommons.org/licenses/by/4.0/

\begin{abstract}
A result from Kieffer, as outlined at the beginning of the article identifies two different candidates for initial time steps, delta t. We assert that this difference in time steps may be related to a specific early universe Lorentz Violation. The author asserts that the existence of early universe Lorentz violation in turn is assisting in a breakup of primordial black holes. And that also has a tie into Kieffer different time steps as outlined. And the wrap up is given in the final part of this document.
\end{abstract}

\section{Keywords}

Kieffer Density Matrix, Initial Time, Lorentz Violations, Black Holes

\section{Introduction, First of All Finding Two Different Initial Time Steps}

Our initial goal is to obtain, via a Kieffer Density function candidate minimum time steps which will be for the purpose of giving input into an uncertainty principle of the form [1] [2] [3] [4]

$$
\Delta E \Delta t \approx 4 \hbar
$$

With a use of [4] [5]

$$
\begin{aligned}
& a(t)=a_{\text {initial }} t^{v} \\
& \Rightarrow \phi=\ln \left(\sqrt{\frac{8 \pi G V_{0}}{v \cdot(3 v-1)}} \cdot t\right)^{\sqrt{\frac{v}{16 \pi G}}}
\end{aligned}
$$




$$
\begin{aligned}
& \Rightarrow \dot{\phi}=\sqrt{\frac{v}{4 \pi G}} \cdot t^{-1} \\
& \Rightarrow \frac{H^{2}}{\dot{\phi}} \approx \sqrt{\frac{4 \pi G}{v}} \cdot t \cdot T^{4} \cdot \frac{(1.66)^{2} \cdot g_{*}}{m_{P}^{2}} \approx 10^{-5}
\end{aligned}
$$

In doing all of this we are making full use of the following from [4] due to a one loop approximation [4] [6]

$$
\rho(\phi, \phi) \approx\left\{\exp \left(\frac{ \pm 3 M_{P}^{4}}{8 \cdot V(\phi)}\right)\right\} \cdot \phi^{-\tilde{Z}-2}
$$

Which after we isolate out $\Delta t$ makes use of Equation (1), which is derived as given in [1] [2] [3] [4]

$$
\Delta E \approx \frac{\hbar \Delta t}{2 \gamma t_{P}^{2}} \cdot \frac{8 \hbar^{2} \gamma t_{P}^{2}}{(\hbar \Delta t)^{2}} \equiv \frac{4 \hbar}{\Delta t}
$$

We will be applying Equation (4) to obtain $\Delta t$, and then from this step applying Equation (1) to say foundational import issues of time flow in the beginning.

1a. Understanding the probability density functional as an Outgrowth of

\section{Kieffer's derivation}

Our assumption is that time, $t$, which becomes $\Delta t$ is extremely small. Hence without loss of generality we write, if as an example, $\tilde{Z} \approx 2$.

And we simplify time dependence by setting $v=\frac{3 \pi}{\sqrt{2}}$ in Equations (2)-(4) Then, without loss of generality, if we observe this, and set $\Theta$ as a probability density value of Equation (4), we then have [4] [6]

$$
\left(\frac{1}{4} \cdot \exp \left( \pm \frac{3}{8} \cdot \frac{M_{P}^{4}}{V_{0}}\right)\right) \cdot\left(1-4 \cdot\left(\sqrt{\frac{8 \pi V_{0}}{\left(\frac{3 \pi}{\sqrt{2}}\right) \cdot\left(\frac{9 \pi}{\sqrt{2}}-1\right)}}\right) \cdot \Delta t\right) \approx \Theta
$$

If so, then we have a minimum time step of the form

$$
\Delta t \approx 4\left(\sqrt{\left.\frac{\left(\frac{3 \pi}{\sqrt{2}}\right) \cdot\left(\frac{9 \pi}{\sqrt{2}}-1\right)}{8 \pi V_{0}}\right)}\right) \cdot\left(1-4 \Theta \exp \left( \pm \frac{3}{8} \cdot \frac{M_{P}^{4}}{V_{0}}\right)\right)
$$

Equations ((6), (9)) are keys to the formation of a Lorentz violation, for the reasons we will go into in the next section.

Now for the existence of an initial Lorentz violation, in part linked to Equation (6).

\section{Making Use of [7] and the Use of Lorentz Violation, as in [7] to Define More Precisely the Contribution of Gravitons to Both DE, Due to the Breakup of Black Holes}

To do this we need to review the Lorentz violating energy-momentum relation- 
ship. In short we have that

$$
E^{2}=p^{2}+m^{2}-\tilde{\lambda} p^{3}
$$

where the positive LV parameter $\tilde{\lambda}$ is usually assumed of the order of Planck mass, $\lambda \sim 1 / M$ (Planck mass). This Lorentz violating energy-momentum relationship leads to, according to [7] [8]

$$
\mathrm{d} \rho=\frac{8 \pi p^{3} c}{h} \cdot \frac{\sqrt{1+\frac{m^{2}}{p^{2}}-\tilde{\lambda} p}}{\exp \left(\left[\frac{c p}{k_{B} T_{\text {temp }}}\right] \cdot \sqrt{1+\frac{m^{2}}{p^{2}}-\tilde{\lambda} p}\right)-1} \cdot \mathrm{d} p
$$

$p \approx 1 / \tilde{\lambda}$ is used if we integrate, Equation (6) and if we use the first order Romberg numerical integration scheme as given in [9], page 695, so then for high temperature

$$
\begin{gathered}
\rho \approx \frac{8 \pi M_{P}^{3} C}{h} \cdot \frac{\sqrt{\frac{m^{2}}{M_{P}^{2}}}}{\exp \left(\left[\frac{c p}{k_{B} T_{\text {temp }}}\right] \cdot \sqrt{\frac{m^{2}}{M_{P}^{2}}}\right)-1} \\
\approx \frac{4 M_{P}^{2} C}{\hbar} \cdot\left(\left[\frac{c m}{k_{B} T_{\text {temp }}}\right]-\frac{1}{2}\left(\frac{c m}{k_{B} T_{\text {temp }}}\right)^{2}\right) \\
\underset{k_{B}=c=\hbar=M_{P}=1}{\longrightarrow} 4 \cdot\left(\left[\frac{m}{T_{\text {temp }}}\right]-\frac{1}{2}\left(\frac{m}{T_{\text {temp }}}\right)^{2}\right)
\end{gathered}
$$

We will then in the next section interpret Equation (9) when we set Notice here that we have restrictions on particle manufacturing for the theory. This is in line with [4]

$$
\Delta E \approx \hbar \cdot\left(\sqrt{\frac{8 \pi V_{0}}{\left(\frac{3 \pi}{\sqrt{2}}\right) \cdot\left(\frac{9 \pi}{\sqrt{2}}-1\right)}}\right) \cdot\left(1+4 \Theta \exp \left( \pm \frac{3}{8} \cdot \frac{M_{P}^{4}}{V_{0}}\right)\right)
$$

Futher more we can make the following identification $m \approx m_{g} \cdot N_{g}$.

\section{Interpreting Equation (13), So as to Ascertain the Number of Gravitons}

We are then looking at [7] [8] Using the Planck units renormalized such that $k_{B}=c=\hbar=M_{P}=1$, we have that in the Pre Planckian state we can look at

$$
\rho \approx 4 \cdot\left(\left[\frac{10^{-65} N_{g}}{\left(T_{\text {temp }} / T_{P}\right)}\right]-\frac{1}{2}\left(\frac{10^{-65} N_{g}}{\left(T_{\text {temp }} / T_{P}\right)}\right)^{2}\right) \approx 10^{-60}
$$

We then can up to a modeling round off make the following approximation This value of Equation (11) will lead to approximately if $\left(T_{\text {temp }} / T_{P}\right) \sim 1$ [9] 


$$
\begin{aligned}
N_{g} & \approx 10^{57} \times\left(1 \pm\left(1-2 \times 10^{-60}\right)\right) \\
& \approx 2 \times 10^{57}-2 \times 10^{-3}
\end{aligned}
$$

We will be utilizing [9] and [10] due to the subtle physics involved. [9] appeals to specific Lorentz transformations, which are necessary to understand the physics of the BEC (Bose-Einstein condensation) assumed in [10] for the treatment of mini black holes as BEC of gravitons. To see BEC invoked, look at [10] which has the specified form as given.

To whit the differences in Equations (9) and (10), will be such that we can now attend to the final piece of the puzzle which is primordial black holes being composed of Gravitons as Bose-Einstein condensates so the following is true, namely [10]

$$
\begin{aligned}
& m \approx \frac{M_{P}}{\sqrt{N_{\text {gravitons }}}} \cdot M_{P} \\
& M_{B H} \approx \sqrt{N_{\text {gravitons }}} \cdot l_{P} \\
& R_{B H} \approx \sqrt{N_{\text {gravitons }}} \\
& S_{B H} \approx k_{B} \cdot N_{\text {gravitons }} \\
& T_{B H} \approx \frac{T_{P}}{\sqrt{N_{\text {gravitons }}}}
\end{aligned}
$$

If, so then we will have applying special relativity, and a further elaboration of Equation (13a) can be done as follows, namely [9]

$$
m \approx \frac{m_{g}}{\sqrt{1-\left(\frac{v_{g}}{c}\right)^{2}}} \approx \frac{M_{P}}{\sqrt{N_{\text {gravitons }}}} \approx 10^{-10} \text { grams }
$$

Given us, let us compare terms, and tell ourselves what we can expect if we have the variance in Equation (14) for BEC treatment of Gravitons.

\section{What $10^{57}$ Gravitons in a Radius of $\mathbf{1 0 0 0}$ Kilometers Means in Terms of DE and a Cosmological Constant Calculation}

We will first of all refer to an early universe treatment of the uncertainty principle is, in the startup of inflationary cosmology [7] [11].

The value of time $t$ will be set as $t \sim\left(10^{-32} \mathrm{~s} / t(\right.$ Planck $\left.)\right)$ whereas we can utilize the ideas of having Planck time set $\sim 5 \times 10^{-44}$ seconds, hence, $t \sim 10^{12}$, in Planck Units, whereas $\hbar=G=\ell_{P}=m_{P}=k_{B}=1$, so then we will have, as reset as [7]

$$
\begin{aligned}
\rho & \approx \frac{\gamma \cdot\left(10^{24}\right)}{8 \pi}+V_{0}\left\{\frac{8 \pi \cdot V_{0} \cdot\left(10^{12}\right)}{\gamma \cdot(3 \gamma-1)}\right\}^{\frac{1}{2} \cdot\left(\sqrt{\frac{\gamma}{\pi}}-4 \cdot \sqrt{\frac{\pi}{\gamma}}\right)} \approx \frac{E_{\text {effective }}}{(1000 \mathrm{~km})^{3}} \\
& \approx \frac{E_{\text {effective }}}{\left(6.25 \times 10^{40}\left(\ell_{P}=1\right)\right)^{3}}
\end{aligned}
$$

The interesting thing, is that the factor of roughly $10^{\wedge}-120$ shows up in this 
situation so as to imply that there may be some linkage between setting the effective energy as roughly some proportional power value of Planck Mass [7].

$$
10^{2} m_{P} \text { (gravitons) } \Rightarrow 10^{67} \text { gravitons per } 10^{5} m_{P} \text { (black hole) }
$$

\section{Making DE Equivalent to a Sea of Initial Gravitons, in Re- gime $10^{-43}$ to $10^{-32}$ Seconds}

Roughly put, one hydrogen atom is about 1.66 times $10^{-24}$ grams. The weight of a Massive graviton is about $10^{-65}$ grams [12], hence we are talking about $10^{-22}$ grams, or about $10^{44}$ gravitons, with each graviton about $6 \times 10^{-32} \mathrm{eV} / \mathrm{c}^{2}$ after $10^{-27}$ seconds, the following in the set of equations given below are Equivalent, and that these together will lead to a cosmological constant, $\Lambda$ of the sort which we will be able to refer to later with 1 graviton $\approx 10^{-65} \mathrm{~g}$. Then set to have a micro sized black hole of the size [7]

$$
M_{P B H}\left(10^{-43}\right) \approx 10^{15} \times\left(\frac{10^{-43} \mathrm{~s}}{10^{-23} \mathrm{~s}}\right) \mathrm{g} \approx 10^{-5} \mathrm{~g} \approx 1 M_{P}
$$

Table 1, inputs into forming DE if one is assuming uniform distribution of $\mathrm{BH}$ to be broken up by Karen Freeze model as given in the following Table 1 .

Assuming that gravitons contribute to the Dark Energy value will lead to us using the Karen Freeze model, with gravitons being released in the early universe by the breakup of early universe black holes which have a maximum value of about $1 \mathrm{~g}$, as opposed to the value of the Sun which has about $10^{33}$ grams, in first $10^{-32}$ seconds.

\section{What $10^{57}$ Gravitons in a Radius of 1000 Kilometers Means if We Go to the Rosen Early Universe Cosmology}

Let us first recall the Shalyt-Margolin and Tregubovich (2004, p.73) [13], ShalytMargolin (2005, p.62) [14] [15].

For sufficiently small $\gamma$. The above could be represented by [3] [14]

$$
\begin{aligned}
& \Delta E \approx \frac{\hbar \Delta t}{2 \gamma t_{P}^{2}} \cdot\left(1 \pm\left(1-\frac{8 \hbar^{2} \gamma t_{P}^{2}}{(\hbar \Delta t)^{2}}\right)\right) \\
& \Rightarrow \Delta E \approx \text { either } \frac{\hbar \Delta t}{2 \gamma t_{P}^{2}} \cdot \frac{8 \hbar^{2} \gamma t_{P}^{2}}{(\hbar \Delta t)^{2}} \text {, or } \frac{\hbar \Delta t}{2 \gamma t_{P}^{2}} \cdot\left(2-\frac{8 \hbar^{2} \gamma t_{P}^{2}}{(\hbar \Delta t)^{2}}\right)
\end{aligned}
$$

Table 1. Summary of infiationary and post inflationary information.

\begin{tabular}{llll}
\hline $\begin{array}{l}\text { Number of black } \\
\text { holes, }\end{array}$ & $\begin{array}{l}\text { Mass of black hole of size } 1 \\
\text { Planck mass set aside for } \\
\text { gravitons }\end{array}$ & $\begin{array}{l}\text { Mass of black hole for } 10^{8} \\
\text { gravitons }\end{array}$ & Radii of proto universe \\
\hline $10^{8}$ & $10^{-8}$ grams & $10^{-5} \mathrm{~g}=1$ Planck mass & 1000 Kilometers \\
$\begin{array}{l}\text { Volume of } \\
\begin{array}{l}\text { Universe is } 10^{3} \\
\text { kilometers, cubed }\end{array}\end{array}$ & $\begin{array}{l}\text { Starting range for Mass of } \\
\text { black hole for Gravitons }\end{array}$ & $\begin{array}{l}\text { Assumed to be starting range } \\
\text { of BH masses, at about } 10^{-43} \\
\text { seconds }\end{array}$ & $\begin{array}{l}\text { constructing black holes which may be torn } \\
\text { asunder by Karen Freeze's criteria }\end{array}$ \\
\hline
\end{tabular}


This would lead to a minimal relationship between change in $E$ and change in time as represented by Equation (18), so that in the end we would write a limiting case as

$$
\Delta E \Delta t \approx 4 \hbar
$$

Having brought this up, let us then go to the Rosen [16] version of comology, and this needs explanation due to its rescaling of the values of the comology time and temperatures involved. The key point of this mini chapter will be to summarize derivation of the space-time temperature [16]

$$
T=\left(\rho_{F} / \sigma\right)^{1 / 4} \cdot \frac{\breve{a} r^{7}}{\left(\breve{a}^{4}+r^{4}\right)^{2}}
$$

With $\breve{a}=10^{-3} \mathrm{~cm},\left(\rho_{F} / \sigma\right)^{1 / 4}=1.574 \times 10^{32} \mathrm{~K}$ (kelvin) Then according to [2], the initial temperature is $T_{\text {initial }}=2.65 \times 10^{-180} \mathrm{~K}$ (kelvin) Whereas the temperature where one has the breakup of Primordial black holes is at $T_{\text {black hole breakup starts }}=7.41 \times 10^{31} \mathrm{~K}$ (kelvin). As given in [2], we have then that if $R$ (radius) is between 1 meter to say 1000 Kilometers this mass $m$ breaks up for $m$ as given by [7] [17]

$$
m \approx \frac{8 \pi R(\text { radius })^{3} \cdot \rho}{3}
$$

Here, the density function is given by Equation (15) and Equation (11), for our application and also we obtain for black holes a break up criteria for mass $\mathrm{m}$ Black holes if [17]

$$
m \approx-\left(\frac{4 \pi \rho}{3}\right) \cdot\left(1+3 \cdot \frac{\rho_{G}}{p_{G}}\right) \cdot \frac{8 m^{3}}{M_{P}^{6}}
$$

So we can have the start of breakup of black holes, if we have gravitons from $1 / 1000$ of the mass of given black holes, and if black holes contribute DE according to when pressure is approximately equal to the negative value of the density which would lead to a Black hole contribution of Equation (23) to DE. As given below [7].

$$
\text { DE from black holes }=7 \times 10^{-30} \mathrm{~g} / \mathrm{cm}^{3}=7 \times 10^{-6} \mathrm{~g} /(1000 \mathrm{~km})
$$

This rough value of DE, as given in Equation (23) will be directly compared to what we can expect as far as applying in Equation (23) as to comment directly on the $\Delta t$ time interval for the active generation of $\mathrm{DE}$ in the early cosmos.

Keep in mind that J. W. Moffat in [18] postulated in the initial phases of cosmology a situation for which we have no conservation of energy, and in fact this is exactly the situation we could be portraying here, that is if the description of the Rosen cosmology as in [16] are not wrong, i.e. we have a massive violation of conservation of energy.

This would be doable if the initial phases of creation of the universe follow [16] if we utilize, initially a near zero temperature start regime in the early universe, as in [16]. The early universe will have an energy input via thermal inputs 
of a value commensurate with

$$
E(\text { thermal energy })=\frac{d(\operatorname{dim}) \cdot k_{B} \cdot T_{\text {universe }}}{2}
$$

\section{What about a Tie into an Early Wavefunction for the Universe Argument? Viutilli Treatment of Schrodinger Equation for Curved Space-Time}

The tie in, as can be stated is to make, after all the proximations, the following argument in [19] [20] we have after several manipulations a wave function dominated by

$$
\Psi \approx \Psi_{\text {initial }} \cdot \exp \left(i \sqrt{\frac{m^{2} c^{2}}{\hbar^{2}}+R_{r}^{r}} \cdot r\right)
$$

Whereas the approximation given is that we will be examining what if $R_{r}^{r}$ is a constant. If so then, using the S.E. will be leading to an effective energy of

$$
\begin{gathered}
E_{\text {eff }} \approx\left[\frac{m c^{2}}{2}+R_{r}^{r} \cdot \frac{\hbar^{2}}{2 m}\right] \\
R_{r}^{r} \approx 12 H_{\text {initial }}^{2}+\frac{6 \kappa}{a_{\text {min }}^{2}} \underset{a_{\min }^{2} \approx 10^{-50}}{\longrightarrow} \frac{6 \kappa}{a_{\text {min }}^{2}}, \text { if } \kappa \neq 0
\end{gathered}
$$

Where we are assuming there is a non zero flatness term.

This will be linkable to, if we are assuming temperature eventually appears,

$$
\begin{aligned}
& E_{\text {eff }} \approx\left[\frac{k_{B} d(\operatorname{dim}) \cdot T_{\text {eff }}}{2}\right] \approx\left[\frac{m c^{2}}{2}+\frac{3 \hbar^{2} \kappa}{a_{\text {min }}^{2} m}\right] \approx \hbar \cdot \omega_{\text {effective }} \\
& \Rightarrow \omega_{\text {effective }} \approx\left[\frac{m c^{2}}{2 \hbar}+\frac{3 \hbar \kappa}{a_{\text {min }}^{2} m}\right] \approx\left[\frac{k_{B} d(\operatorname{dim}) T_{\text {eff }}}{2 \hbar}\right]
\end{aligned}
$$

The final step is to assume a non zero effective temperature we are in this situation directly using the idea of Lorenz invariance being broken in order to make sense of what Equation (28) is stating, we can also look at the following' heuristic short hand, namely of Equations (13)-(14) being employed for the value of $\mathrm{m}$ above so as to have, say we are considering black holes of BEC configuration

$$
m \approx \frac{2 \hbar \cdot \omega_{\text {eff }}}{c^{2}} \cdot\left[1 \pm \sqrt{1-\frac{6 \kappa c^{2}}{a_{\min }^{2} \cdot \omega_{\text {eff }}^{2}}}\right] \approx \frac{M_{P}}{\sqrt{N_{g}}}
$$

In such a situation, for gravitons being released from black holes due to a BEC condensate for black holes, as being destroyed by the Karen Freeze criteria, we must realize that a Lorentz violation would either tend to favor a very small release of gravitons being put into Equation (26) or a large value i.e., the Lorentz violation would then be favoring the term $N_{g}$ as being favored by the larger value as given in Equations (12)-(14). This leads to the following question to be raised. Assume we could release a breakup of primordial Black holes, do we 
have, say any conditions where.

After setting up the formulation of conditions for the creation of DE according to which we are looking at in the regime of space-time from less than a meter in radii to $1000 \mathrm{~km}$ in radii we have the formation of DE with the given frequency, for setting up DE, and then by default the Cosmological constant,

$$
V(\phi(t)) \approx \omega_{\text {Graviton generated DE }}
$$

Is Equation (30) in any way commensurate with the Frequency stated as of Equation (28) and is this identification due to an early Universe Lorentz violation?

$$
\begin{gathered}
c \rho_{d S}=\left.\left.\left.\frac{\left(R_{00}-g_{00} R\right)}{8 \pi}\right|_{d S}\right|_{d S} \approx \frac{\left(-3 \frac{\ddot{a}}{a}-g_{00} 6 \Lambda\right)}{8 \pi}\right|_{d S}
\end{gathered}
$$

See this final take away as to what the cosmological constant is equivalent to, i.e. does the following make sense? If we multiply Equation (19) say by the cube of a Planck volume, and then can we work with the following derivation? One solution is as follows, [21] [22]

$$
\Delta E \Delta t \approx \hbar \equiv \hbar \omega \Delta t \approx \hbar \omega \cdot\left(\frac{2}{3 a_{\min }}\right)^{1 / \gamma} \Rightarrow \omega \approx \hbar^{-1} \cdot\left(\frac{2}{3 a_{\min }}\right)^{-1 / \gamma}
$$

The above is one such relationship. We need to reconcile it with Equation (32) in which we have the following provided that we have, at the point where $\kappa=0$ (flatness) will lead to $m$ not equal to

$$
\begin{gathered}
\kappa=0 \text { (flatness) } \Rightarrow N_{g} \approx \frac{10^{50 / \gamma}}{(3 / 2)^{2 / \gamma}} \\
m \neq N_{g} \cdot M_{P} \text { if } \kappa=0, \text { but instead } m \approx \frac{M_{P}}{\sqrt{N_{g}}}
\end{gathered}
$$

What is set in Equation (19) is in line with also using

$$
a(t)=a_{\min } t^{\gamma}
$$

Leading to [21] [22] the inflaton

$$
\phi \approx \sqrt{\frac{\gamma}{4 \pi G}} \ln \left\{\sqrt{\frac{8 \pi G}{\gamma \cdot(3 \gamma-1)}} \cdot t\right\}
$$

And what we will use later the "inflaton potential" we write as [21] [22]

$$
V=V_{0} \cdot\left\{\sqrt{\frac{8 \pi G V_{0}}{\gamma(3 \gamma-1)}} \cdot t\right\}^{\sqrt{\frac{\gamma}{4 \pi G}}-\sqrt{\frac{8 \pi G}{\gamma}}}
$$


Furthermore, we have from applying a reference from the author, [21] and [22], using pages 212-213 of reference [22]

$$
m \cdot \partial_{t}\left(a^{2}-a^{3}\right)=0
$$

And then, a minimum time step we define via a minimum time step of

$$
t=\left(\frac{2}{3 a_{\min }}\right)^{1 / \gamma}
$$

Note that if the time as defined by Equation (39) is on the order of Planck time, i.e. $10^{-44}$ seconds, we have then that $\gamma \approx 61-62$ Given this value of 61 to 62 in gamma, it leads to having $N_{g}$ approximately of the value of 10 which is in line with, if $\mathrm{m}$ is in this case commensurate with Equation (14), i.e. an effective graviton mass. Furthermore gamma of the value of 61 or so would lead to an effective frequency $1.8549 \times 10^{43} \mathrm{~s}^{-1}$ times $1 /$ sqrt of 10. i.e. $\mathrm{c} /$ Planck Length times $1 /$ sqrt of 10, which is normalized in Planck Units to be $\sim 1 /$ sqrt of 10 .

In line with Equation (25), and normalized Planck units, this would correspond to $1.416784(16) \times 10^{32} \mathrm{~K} /$ sqrt 10 , or about a breakup of Planck sized black holes at a temperature, due to Lorentz violation processes, early on of

$$
T_{\text {Plank BH Breakup }} \approx T_{P} / \sqrt{10} \equiv 0.507 \times 10^{20} \mathrm{GeV}
$$

Having this temperature for the energy is then equivalent, if we have a transition from curved to flat space to writing, if Gamma is $\sim 61$ and we have Planck time, after a Lorentz violating transformation from curved space to Flat space which we would write as

$$
\begin{aligned}
& V=V_{0} \cdot\left\{\sqrt{\frac{8 \pi G V_{0}}{\gamma(3 \gamma-1)}} \cdot t\right\}^{\sqrt{\frac{\gamma}{4 \pi G}}-\sqrt{\frac{8 \pi G}{\gamma}}} \\
& \underset{l_{\text {Planck }=\hbar \equiv G=1}}{\longrightarrow} V_{0} \cdot\left\{\sqrt{\frac{8 \pi V_{0}}{\gamma(3 \gamma-1)}} \cdot t\right\}^{\sqrt{\frac{\gamma}{4 \pi}}-\sqrt{\frac{8 \pi}{\gamma}}} \approx \omega \propto\left(\frac{2}{3 a_{\text {min }}}\right)^{-1 / \gamma}
\end{aligned}
$$

Which is in turn related to [7]

$$
\begin{gathered}
P_{G}=\frac{\gamma \cdot t^{2}}{8 \pi G}-V_{0} \cdot\left\{\frac{\sqrt{8 \pi G V_{0}}}{\gamma \cdot(3 \gamma-1)} \cdot t\right\}^{\sqrt{\frac{\gamma}{4 \pi G}}-\sqrt{\frac{8 \pi G}{\gamma}}} \\
\rho_{B H}=\frac{m_{p}^{4}}{32 \pi} \cdot\left(\frac{m_{P}}{m}\right)^{4} \cdot \frac{1}{\left|1+3 \frac{P_{G}}{\rho_{G}}\right|}
\end{gathered}
$$

What we will be looking at would be having a glimpse of

$$
\frac{P_{G}}{\rho_{G}} \approx \frac{\frac{\gamma \cdot t^{2}}{8 \pi G}-V_{0} \cdot\left\{\frac{\sqrt{8 \pi G V_{0}}}{\gamma \cdot(3 \gamma-1)} \cdot t\right\}^{\sqrt{\frac{\gamma}{4 \pi G}}-\sqrt{\frac{8 \pi G}{\gamma}}}}{\frac{\gamma \cdot t^{2}}{8 \pi G}+V_{0} \cdot\left\{\frac{\sqrt{8 \pi G V_{0}}}{\gamma \cdot(3 \gamma-1)} \cdot t\right\}^{\sqrt{\frac{\gamma}{4 \pi G}}-\sqrt{\frac{8 \pi G}{\gamma}}}}
$$


Doing this would be at a minimum of having, a distance greater than or equal to

$$
\lambda_{D E} \approx 10^{30} \ell_{P}
$$

Assume then we have, for the sake of argument

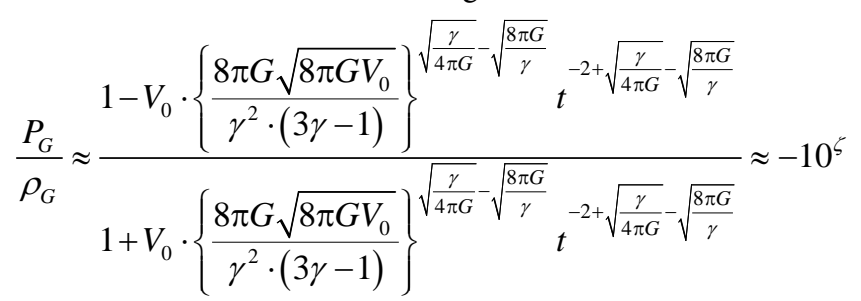

The particulars of the coefficient of the right hand side of Equation (46), if $\hbar=G=\ell_{P}=t_{P}=1$, then if we set

$$
\frac{\gamma}{4 \pi}-2 \sqrt{\frac{\gamma}{4 \pi}}-1=0
$$

So then we have if we wish to neutralize sensitivity to time itself at first approximation

$$
\gamma=4 \pi \cdot(1 \pm \sqrt{2})^{2}
$$

We can then look at the following

$$
\frac{1-V_{0} \cdot\left\{\frac{8 \pi \sqrt{8 \pi V_{0}}}{16 \pi^{2} \cdot(1 \pm \sqrt{2})^{4} \cdot\left(12 \pi \cdot(1 \pm \sqrt{2})^{2}-1\right)}\right\}^{(1 \pm \sqrt{2})-\frac{\sqrt{2}}{(1 \pm \sqrt{2})}}}{1+V_{0} \cdot\left\{\frac{8 \pi \sqrt{8 \pi V_{0}}}{16 \pi^{2} \cdot(1 \pm \sqrt{2})^{4} \cdot\left(12 \pi \cdot(1 \pm \sqrt{2})^{2}-1\right)}\right\}^{(1 \pm \sqrt{2})-\frac{\sqrt{2}}{(1 \pm \sqrt{2})}} \approx-10^{\zeta}}
$$

The above equation can be used, to locate appropriate values for $V_{0}$ in units where

$$
\hbar=G=\ell_{P}=t_{P}=m_{P}=c=1
$$

Given an approximate value for $V_{0}$ we will then proceed to come up with examining

$$
\begin{aligned}
& \rho_{B H}=\frac{m_{p}^{4}}{32 \pi} \cdot\left(\frac{m_{P}}{m}\right)^{4} \cdot \frac{1}{\left|1-3 \cdot 10^{-\zeta}\right|} \\
& M_{\text {black hole primordial }} \sim 10^{15} \times\left(\frac{t}{10^{-23} \mathrm{~s}}\right) \mathrm{g}(\text { grams })
\end{aligned}
$$

The Lorentz symmetry breaking would be occurring at roughly a multiple of Planck time $t$, at a distance of say where we would be using we will first start off with the redone calculation as to the vacuum energy and how we rescale them to be in sync as to the observed value for vacuum energy which is of the present era. This methodology is consistent with the zero-point energy calculation, we 
start off with the following as given by [23] [24] [25]

$$
\begin{aligned}
& \frac{1}{2} \cdot \sum_{i} \omega_{i} \equiv V(\text { volume }) \cdot \int_{0}^{\hat{\lambda}} \sqrt{k^{2}+m^{2}} \frac{k^{2} \mathrm{~d} k}{4 \pi^{2}} \approx \frac{\hat{\lambda}^{4}}{16 \pi^{2}} \\
& \underset{\hat{\lambda}=M_{\text {Planck }}}{\longrightarrow} \rho_{\text {boson }} \approx 2 \times 10^{71} \mathrm{GeV}^{4} \approx 10^{119} \cdot\left(\rho_{D E}=\frac{\Lambda}{8 \pi G}\right)
\end{aligned}
$$

In stating this we have to consider that $\rho_{D E}=\frac{\Lambda}{8 \pi G} \approx \hbar \cdot \frac{(2 \pi)^{4}}{\lambda_{D E}^{4}}$, so then that the equation we have to consider is a wavelength $\lambda_{D E} \approx 10^{30} \ell_{\text {Planck }}$ which is about $10^{30}$ times a Plank length radius of a space-time bubble. That would, we have after $10^{-42}$ seconds

$$
\rho_{D E}=\frac{\Lambda}{8 \pi G} \approx \hbar \cdot \frac{(2 \pi)^{4}}{\lambda_{D E}^{4}}
$$

We then have to consider how to reach the experimental conditions for when a nonsingular expansion point for Cosmology, will after $10^{-42}$ seconds lead to Equation (4). That means a discussion of what Rosen and Israelit did in [16]. Our point in this admittedly ad hoc discussion will be to do dimensional analysis when applying [16] is that we have a factor of $10^{30}$ expansion as to where we can at least measure the onset of DE.

Looking now at Rosen and Israelit, in terms of Thermodynamics of a non-singular universe [16] will be relevant for several reasons

A. We will be able to come up with an initial temperature of $10^{-180} \mathrm{Kelvin}$, at a radius of about Planck length, in value. Almost absolute zero B. The temperature of space-time will be of the order of Planck Temperature after expansion of about $10^{30}$ times from the initial nonsingular configuration earliest phase, whereas we have the following Entropy value of [26]

$$
S \sim 3 \cdot\left[1.66 \sqrt{g_{*}}\right]^{2} T^{3}
$$

Here, the degrees of freedom term is defined in [16] and initially is about 110 This maximum entropy for slightly less than a Planck sized temperature, $T$ occurs at the point where we have maximum Graviton release, due to the breakup of black holes as postulated by Karen Freeze, as when the space-time density is in line with Equation (60) with we wish to refer to the following as motivation in order to link this to graviton mass and other such concerning heavy gravity, so we have the following, as to how to obtain the mass of an inflaton, namely use, if Equation (37) is used for $V$ (phi)

$$
\begin{aligned}
& m_{\mathrm{inf}}^{2}=\left.\frac{\mathrm{d}^{2} V(\phi)}{\mathrm{d} \phi^{2}}\right|_{\phi=0, t=3.9776 \cdot t_{P}}=\frac{16 \pi}{3 \pi / \sqrt{2}} \\
& \Rightarrow m_{\mathrm{inf}}=2.74635619187 \text { (Planck units) }
\end{aligned}
$$

IMO the inflaton mass is 2.746356 times Planck Mass, and this is a starting value of inflaton mass at $t=3.9776$ Planck time. We should compare the inter relationship of this inflaton mass, in a volume of space with the results of Equation (50). The interrelationship of this inflaton mass, at a given time $t \sim 3.997$ 
Planck time, as compared Equation (50) for the breakup of black holes, and with the information given in Equations (8) and (9) of this document.

\section{Conflicts of Interest}

The author declares no conflicts of interest regarding the publication of this paper.

\section{References}

[1] Shalyt-Margolin, A.E. (2006) Deformed Density Matrix and 21 Quantum Entropy of the Black Hole. Entropy, 8, 31-43. https://doi.org/10.3390/e8010031

[2] Narlinkar, J.V. (1982) Quantum Fluctuations near the Classical Space-Time Singularity. Proceedings of the Workshop on Gravitation and Relativistic Astrophysics, Ahmedabhad, 18-20 January 1982, 135-151.

[3] Shalyt-Margolin, A.E. (2005) The Density Matrix Deformation in Physics of the Early Universe and Some of Its Implications. In: Reimer, A., Eds, Quantum Cosmology Research Trends, Horizons in World Physics, Vol. 246, Nova Science Publishers, Inc., Hauppauge, Chapter 2, 49-91.

[4] Beckwith, A. (2021) Using Kiefer Density Matrix for Time Flow Analysis and How This Links to a Proof of Production of Planck Mass BHs during Inflation and Their Resulting Breakup, Leading to a DE Candidate. Journal of High Energy Physics, Gravitation and Cosmology, 7, 1005-1018. https://doi.org/10.4236/jhepgc.2021.73059

[5] Padmanabhan, T. (2006) World Scientific Series in Astronomy and Astrophysics: An Invitation to Astrophysics. Vol. 8, World Press Scientific, Singapore. https://doi.org/10.1142/6010

[6] Kiefer, C. (2000) Conceptual Issues in Quantum Cosmology. In: Kowalski-Glikman, J., Ed., Toward Quantum Gravity, Vol. 541, Springer-Verlag, New York, 158-187. https://doi.org/10.1007/3-540-46634-7_7

[7] Beckwith, A. (2021) Using Lorentz Violation for Early Universe GW Generation Due to Black Hole Destruction in the Early Universe as by Freeze. Journal of High Energy Physics, Gravitation and Cosmology, 7, 993-1004. https://doi.org/10.4236/jhepgc.2021.73058

[8] Salesi, G. (2012) Lorentz-Violating Dynamics in the Pre-Planckian Universe. Physical Review D, 85, Article ID: 063502. https://arxiv.org/abs/1110.2234 https://doi.org/10.1103/PhysRevD.85.063502

[9] Carroll, S.M. (2004) Spacetime and Geometry: An Introduction to General Relativity. Illustrated Edition, Addison Wesley, 22.

[10] Chvanis, P.H. (2014) Self Gravitating Bose-Einstein Condensates. In: Calmet, X., Ed., Quantum Aspects of Black Holes, Vol. 178, Springer Verlag, Cham, 151-194. https://doi.org/10.1007/978-3-319-10852-0_6

[11] Padmandabhan, T. (2005) Advanced Topics in Cosmology: A Pedagogical Introduction. https://arxiv.org/abs/astro-ph/0602117

[12] Vainshtein, A.I. (1972) To the Problem of Nonvanishing Gravitation Mass. Physics Letters B, 39, 393-394. https://doi.org/10.1016/0370-2693(72)90147-5

[13] Shalyt-Margolin, A.E. (2004) The Universe as a Nonuniform Lattice in Finite Volume Hypercube.I.Fundamental Definitions and Particular Features. International Journal of Modern Physics D, arXiv: hep-th/0312312. 
[14] Shalyt-Margolin, A.E. (2005) The Density Matrix Deformation in Physics of the Early Universe and Some of Its Implications. Quantum Cosmology Research Trends. Horizons in World Physics, 246, 49-91.

[15] Shalyt-Margolin, A.E. and Tregubovich, A.Y. (2004) Deformed Density Matrix and Generalized Uncertainty Relation in Thermodynamics. Modern Physics Letters A, 19, 71-81. https://doi.org/10.1142/S0217732304012812

[16] Rosen, N. and Israelit, M. (1991) A Simple Model of the Universe without Singularities. In: Zichichi, A., de Sabbata, V. and Sánchez, N., Eds., Gravitation and Modern Cosmology, Springer, Boston, 151-156.

https://doi.org/10.1007/978-1-4899-0620-5_14

[17] Freeze, K., Brown, M. and Kinney, W. (2012) The Phantom Bounce: A New Proposal for an Oscillating Cosmology. In: Mercini-Houghton, L. and Vaas, R., Eds., The Arrows of Time: A Debate in Cosmology, Vol. 172, Springer, Heidelberg, 149-156. https://doi.org/10.1007/978-3-642-23259-6_7

[18] Moffat, J.W. (2010) Lorentz Violation of Quantum Gravity. Classical and Quantum Gravity, 27, Article ID: 135016. https://arxiv.org/abs/0905.1668 https://doi.org/10.1088/0264-9381/27/13/135016

[19] Vuille, C. (2020) Schrödinger's Equation in General Relativity. Journal of Mathematical Physics, 41, 5256-5261. https://doi.org/10.1063/1.533406

[20] Beckwith, A.W. (2017) Examination of Schrodinger Equation in Pre-Planckian Space-Time Early Universe. Journal of High Energy Physics, Gravitation and Cosmology, 3, 21-28. https://doi.org/10.4236/jhepgc.2017.31004

[21] Beckwith, A. (2020) We Begin with a "Trivial" Condition on Massive Gravitons, and Use That to Examine Nonsingular Starts to Inflation, for GW, with GW Strength and Possible Polarization States? Journal of High Energy Physics, Gravitation and Cosmology, 6, 692-709. https://doi.org/10.4236/jhepgc.2020.64048

[22] Tolley, A.J. (2015) Cosmological Applications of Massive Gravity. In: Papantonopoulos, E., Ed., Modifications of Einstein's Theory of Gravity at Large Distances, Vol. 892, Springer Verlag, Cham, 203-224.

https://doi.org/10.1007/978-3-319-10070-8_8

[23] Beckwith, A. (2021) A Solution of the Cosmological Constant and DE Using Breakup of Primordial Black Holes, via a Criteria Brought Up by Dr. Freeze Which Initiates DE as Linked to Inflation. Preprints, Article ID: 2021040210.

[24] Beckwith, A. (2021) A Solution of the Cosmological Constant, Using Multiverse Version of Penrose CCC Cosmology, and Enhanced Quantization Compared. Preprints, Article ID: 2021020208.

[25] Li, M., Li, X.-D., Wang, S. and Wang, Y. (2015) Dark Energy. Peking University-World Scientific Advanced Physics Series Vol. 1, World Scientific, Singapore. https://doi.org/10.1142/9293

[26] Kolb, E. and Turner, M. (1990) The Early Universe (Frontiers in Physics). Addison and Westley, Redwood City, California, USA. 